\title{
Interference Shaping for Improved Quality of Experience for Real-Time Video Streaming
}

\author{
Sarabjot Singh, Student Member, IEEE, Jeffrey G. Andrews, Senior Member, IEEE, \\ and Gustavo de Veciana, Fellow, IEEE
}

\begin{abstract}
The unpredictability of the wireless medium poses a major challenge to delivering a high quality of experience $(\mathrm{QoE})$ for real-time video services. Bursty co-channel interference is a prominent cause of wireless throughput variability, which leads to video QoE degradation, even for a fixed average channel quality. In this paper, we propose and analyze a network-level resource management algorithm termed interference shaping to smooth out the throughput variations (and hence improve the QoE) of video users by decreasing the peak rate of co-channel best effort users. Wireless link capacity variations are mapped to the realtime video packet loss rate, and the interference shaping QoE gain for video users is quantified by benchmarking against a modified multi-scale structural similarity (H-MS-SSIM) index. H-MS-SSIM is an accurate perceptual video quality metric that incorporates the important hysteresis effect whereby the current QoE (which is subjective) may strongly depend on the recent past. The proposed technique increases mean $\mathrm{QoE}$ and reduces the QoE variability over time, with a net perceptual increase of about 2-3x in illustrative settings while incurring insignificant decrease in the QoE for co-channel best effort users. Interference shaping can be implemented in both unicast and multicast real-time video streaming with much higher potential gains for multicast.
\end{abstract}

Index Terms-Quality of experience; resource allocation and interference management; cross-layer design; bursty interference, heterogeneous networks.

\section{INTRODUCTION}

Mobile video traffic is growing at an immense rate with the volume expected to double every year between 2010 and 2015 [1]. The study [1] argues that the availability of powerful mobile wireless devices has propelled the growth of mobile video traffic with the projected share estimated to be twothirds of the total mobile traffic by 2015. Hence, mobile video services would likely be a significant source of revenues for network operators. Besides being bandwidth intensive, live video streaming and conversational services are highly sensitive to delay, jitter, and packet losses. User satisfaction in communication networks is receiving increased interest both from academia and industry as opposed to traditional performance metrics like rate or delay. This is driven by the fact that users churn as they become dissatisfied and hence providers and operators are competing to provide higher QoE with their existing network capacity [2]. As per the ITU, QoE is defined as the overall acceptability of an application or

Manuscript received August 20, 2011; revised January 30, 2012; accepted April 1, 2012. This work has been supported by the Intel-Cisco Video Aware Wireless Networks (VAWN) Program.

The authors are with WNCG, Dept. of Electrical and Computer Engineering at the University of Texas, Austin (email: sarabjot@utexas.edu, $\{$ jandrews,gustavo $\} @$ ece.utexas.edu) service, as perceived subjectively by the end-user, [3]. Hence, it cannot be reliably predicted by traditional network metrics like throughput, jitter or delay and is instead measured in terms of Mean Opinion Score (MOS) (Table II) or Differential MOS (DMOS).

High variability of the wireless video link throughput contributes to significant packet delay variations or jitter in video user's traffic. A jitter buffer at video receiver may be used to counter these variations for stored video delivery. But, for real-time video and teleconferencing applications where user experience is heavily dependent on the time lag and continuous play back, the losses due to packet delays and jitter are unavoidable [4]. Throughput variations in the network lead to higher video quality variations which are regarded as leading to lower end user QoE [5][6][7]. This was reconfirmed in [8] where it was shown that the average quality of the complete video session is often not the correct metric of user's QoE due to hysteresis effect in which the current QoE of a user is negatively influenced by drastic changes in video quality in the recent past.

Current wireless networks pose an interesting challenge to meeting the anticipated future demand of video with satisfactory QoE levels due to their application agnostic design and network architecture. As noted in [9], the evolving 4G wireless standards, which promise less latency and higher throughput, can actually aggravate the problem of wireless video delivery with throughput variations of the order of $90 \%$ on the downlink. With the advent of multi-tiered cellular architectures [10] to meet the growing capacity demand, the number of users being served per cell are shrinking. The small cell base stations are, thus, sporadically active and contribute to bursty interference. Hence, the problem of throughput variation will significantly worsen as networks become more heterogeneous over the next several years.

\section{A. Related Work}

Prior work incorporating temporal quality variations into algorithm design for wireless networks has focussed primarily on intelligent radio resource management from a single base station. Temporal quality fluctuations were incorporated in utility function for resource allocation in [11]. A general network utilization maximization framework to incorporate the variations in utility functions was proposed in [12]. Prioritizing video traffic over best effort traffic has been the theme of many earlier resource management literature, see for e.g. [13],[14]. However, the presented work is in the relatively unexplored 


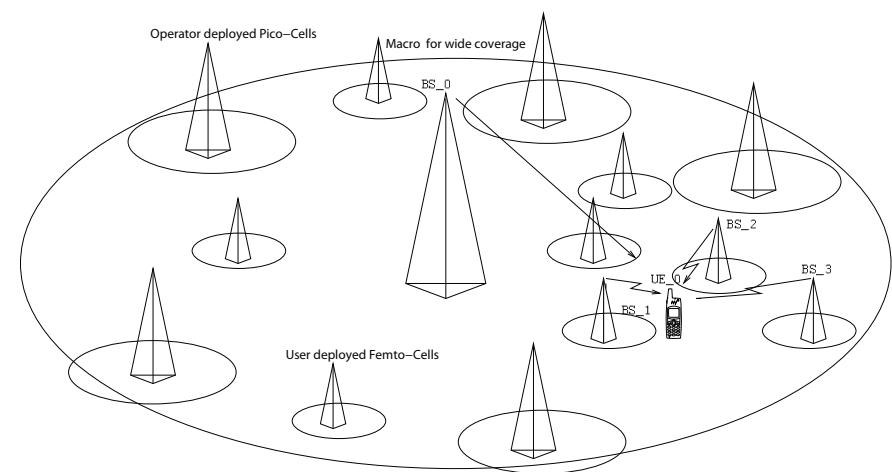

Fig. 1. Model example with macro cell user engaged in real-time video transmission and the small cell users engaged in sporadic web access.

area of prioritizing real-time video traffic over best effort traffic across base stations (BSs).

Most downlink interference avoidance techniques operate in the general theme of reducing the interference power via orthogonal transmission either in time, frequency or space (multi-antenna communications) without affecting the transmit power profile (hence the activity factor) of the interfering base stations. Hence, prior work decreases the received interference power but does not affect the burstiness of interference which in turn depends on the burstiness of transmit power at the interfering base station.

\section{B. Contributions}

Our work ventures into a relatively unexplored area of resource allocation across base stations and presents a QoEaware approach toward designing transmission schemes. We propose an interference shaping algorithm which shapes the received interference power so as to decrease the variation in throughput ${ }^{1}$. for video users by smoothing the transmit power profile (and hence rate) of the interfering BSs. Interference shaping is considerably different from interference reduction and other forms of interference management both in behavior and intent. Behaviorally, rather than mitigating average interference power, the proposed algorithm spreads it in time. The aim of the interference shaping is to reduce the burstiness (second moment) of interference power caused by the bursty traffic at interfering BS whereas the focus of interference management is on reducing the mean interference power (first moment). Thus, the presented algorithm tries to achieve better QoE by shaping (temporally smoothing) the video link throughput. Interference shaping would be useful in scenarios where the link is not capacity-constrained or the interference power is less than the average signal power and hence aimed to complement existing interference mitigation algorithms.

The increase in video QoE due to shaping the interference is evaluated using the hysteresis based temporal pooling strategy for frame based objective quality scores proposed in [8]. The objective full reference multiscale structural similarity (MSSSIM) index [15] is used for benchmarking the improvement

\footnotetext{
${ }^{1}$ Throughput and capacity are used interchangeably in the paper.
}

in quality. We also assess performance improvement using the coefficient of quality variation (CoVQ) metric which measures the standard deviation of quality with respect to its mean. Thus, our conclusions are more credible from a QoE perspective compared to those based on average quality. We also evaluate the decrease in QoE of best effort data users due to transmit power (and hence rate) smoothing by their respective BSs. To the best of our knowledge this is the first work to use the recently developed Weber-Fechner Law (WFL) based framework proposed for web QoE modeling in [16] and [17] for accessing the impact of a network algorithm. WFL quantifies the response of human sensory system to an external stimulus. In this paper, it is shown that in the presence of a single dominant bursty interferer and with interference as the major source of throughput variation, the QoE of real-time video users in the network can be increased by 2-3 times with negligible decrease in QoE for best effort users. In the presence of multiple interfering BSs the gains are comparatively less ( $\sim 1.5$ times), since the total interference is less bursty. The presented simple algorithm can be applied to both unicast and multicast real-time video streaming with gains proportional to the number of video users sharing the same broadcast and interferers in the latter.

A video-specific cellular model with randomly located interfering BSs transmitting bursty best-effort data is also developed. Based on the model, it is shown that aside from smoothing the video link, interference shaping may also increase the mean capacity in scenarios where each interfering BS serves a small number of users. This allows a higher video encoding rate and hence results in higher quality. However, this additional advantage of interference shaping is lost in scenarios where interfering BSs serve a larger population.

The rest of the paper is organized as follows. Sec. II describes the wireless cellular model, various traffic profiles at interfering BSs and a framework for packet losses due to variable throughput. Interference shaping and its effect on packet losses is discussed in Sec. III for unicast as well as multicast streaming. A general video specific cellular model and implications of interference shaping on mean capacity of a typical video user is analyzed in Sec. III-C. In Sec. IV the effect of the proposed approach on QoE is investigated in detail. The implementation details of interference shaping are presented in Sec. V. Sec. VI concludes the paper and suggests future work.

\section{SySTEM MOdEL}

\section{A. Cellular Model}

Consider a setup where $|J|$ BSs transmitting bursty data interfere with a particular video client, $\mathrm{UE}_{0} \cdot \mathrm{BS}_{0}$ is assumed to be serving $\mathrm{UE}_{0}$. The average received Signal to Noise Ratio (SNR) at $\mathrm{UE}_{0}$ from $\mathrm{BS}_{0}$ is assumed to be $\gamma$ and the Interference to Noise ratio (INR) due to $\mathrm{BS} j$ (or $\mathrm{BS}_{j}$ ) is denoted to be $I_{j}$. The bursty interference leads to a bursty Signal to Interference plus Noise Ratio (SINR) at $\mathrm{UE}_{0}$, given by

$$
\mathrm{SINR}=\frac{h_{0} \gamma}{1+\sum_{j=1}^{|J|} I_{j} \mathbf{1}_{j}},
$$


where $\mathbf{1}_{j}$ is the indicator of the event that $\mathrm{BS}_{j}$ is transmitting and $h_{0}$ denotes the channel gain from the $\mathrm{BS}_{0}$ to $\mathrm{UE}_{0}$.

Definition 1. Activity factor $\left(A_{f, j}\right)$ : The fraction of the time a BS is active (or transmitting) is referred to as its activity factor, $A_{f, j}=\mathbb{P}\left(\mathbf{1}_{j}=1\right)$.

An example setup is shown in Fig. 1, where the macro base station $\mathrm{BS}_{0}$ serves the macro user $\mathrm{UE}_{0}$ streaming realtime video (or video telephony) and nearby small cells (e.g. femtocells, picocells) wish to use the same spectrum and hence act as co-channel interferers. A scenario where the co-channel users are interested in web browsing and other best effort applications is considered. This notion can be easily extended to OFDMA systems where users allocated the same set of subcarriers in neighboring cells act as interferers. Voice activity, which requires a low bit rate, is ignored in the analysis, but could be considered in future work.

The transmit power of a base station and the average transmit rate are closely coupled, e.g. Shannon's capacity theorem [18], $C=\log (1+\mathrm{SINR})$. In real wireless systems, like third generation partnership project (3GPP) long term evolution (LTE), this coupling manifests in the form of the transport block size (TBS), the number of bits transmitted in a LTE subframe (14 OFDMA symbols, $1 \mathrm{~ms}$ ), being directly proportional to the power allocated to the corresponding subcarrier group [19]. Let the average rate at which $\mathrm{BS}_{j}$ can transmit (at peak transmit power) be denoted by $R_{o j}$. Let the transmit power be scaled so that the transmit rate of $\mathrm{BS}_{j}$ is lowered by a factor of $\alpha_{j}$ i.e. from $R_{j}(1)=R_{o j}$ to $R_{j}\left(\alpha_{j}\right)=\alpha_{j} R_{o j}$. Getting a closed-form expression in the corresponding required decrease of the average received SINR in terms of the specified system parameters and under general fading distribution is quite complex. Hence to simplify the analysis, for the time being, we resort to the popular Wyner model [20] for average SINR, where channel gains are assumed to be fixed. Since we are interested in the average rate over the time scales of the bursty traffic (which are much larger than the fast fading time scales), the averaging approximation might be reasonable [21]. We emphasize that this assumption is only for computing the average received SINR. With this simplification, the average received SINR at factor of $\alpha_{j}$, $\gamma_{F j}\left(\alpha_{j}\right)$, would satisfy

$$
\begin{aligned}
\alpha_{j} \log _{2}\left(1+\gamma_{F j}(1)\right) & =\log _{2}\left(1+\gamma_{F j}\left(\alpha_{j}\right)\right) \\
\text { or, } \gamma_{F j}\left(\alpha_{j}\right) & =\left(1+\gamma_{F j}(1)\right)^{\alpha_{j}}-1,
\end{aligned}
$$

for all $j=1, \ldots,|J|$. For notational brevity, $\gamma_{F j} \triangleq \gamma_{F j}(1)$.

Definition 2. Rate Scaling Factor $(R S F)(\alpha)$ is a scalar value between 0 and 1 which is used to donate the linear reduction in rate achieved from reducing the transmit power.

A similar notion of power scaling factor $(\beta)$ can be described, with RSF being a function of PSF. Linear scaling of rate (hence non-linear scaling of power) is used for explanation as it lends to better exposition regarding the effect of power scaling on the activity factor $\left(A_{f}\right)$ of the corresponding BS. The INR at the video user due to $\mathrm{BS}_{j}$ can be expressed in terms of its relative strength with respect to the SINR at $\mathrm{BS}_{j}$ 's user $\left(\mathrm{UE}_{j}\right)$ as $I_{j}\left(\alpha_{j}\right)=h_{j} \gamma_{F j}\left(\alpha_{j}\right)$. The mean of the variable $h_{j}\left(\bar{h}_{j}\right)$ captures the proximity ${ }^{2}$ of $\mathrm{UE}_{0}$ to $\mathrm{BS}_{j}$ with respect to $\mathrm{UE}_{j}$. Thus, the SINR at $\mathrm{UE}_{0}$ as a function of RSFs of interfering BSs can be expressed as

$$
\operatorname{SINR}\left(\alpha_{1}, \ldots, \alpha_{j}\right)=\frac{h_{0} \gamma}{1+\sum_{j=1}^{|J|}\left(h_{j}\left(\left(1+\gamma_{F j}\right)^{\alpha_{j}}-1\right)\right) \mathbf{1}_{j}} .
$$

The variation of the interfering probability of the $j^{\text {th }}$ interfering BS or $A_{f, j}$ with RSF, $\alpha_{j}$, depends on its data arrival model, which is discussed next.

\section{B. Traffic Characteristics at $B S_{j}$}

Two types of traffic models are used in this paper to model different usage scenarios served by the interfering BS.

1) Closed-loop traffic model: This model aims to characterize the behavior of a single user's web activity, where increase in service rate leads to increase in user's request rate. The user requests pages of random sizes and the "think times" or interpage request times, are also random. The user's think time is assumed to be exponentially distributed with mean $\frac{1}{\Lambda_{t}}$ and the service time is assumed to be exponentially distributed with mean $\frac{1}{\mu R\left(\alpha_{j}\right)}$. This is the well studied "mainframe/terminal" model [22]. The steady state probability that the transmitter is busy, i.e. the user is not in the thinking phase, can be calculated as

$$
A_{f, j}=\mathbb{E}\left[\mathbf{1}_{j}\left(\alpha_{j}\right)\right]=\frac{\Lambda_{t}}{\mu R\left(\alpha_{j}\right)+\Lambda_{t}}=\frac{\rho_{j}^{c}(1)}{\alpha_{j}+\rho_{j}^{c}(1)},
$$

where $\rho_{j}^{c}(\alpha)=\frac{\Lambda_{t}}{\mu R\left(\alpha_{j}\right)}$. Note that scaling the transmit power and hence the transmit rate would also reduce the rate of user requests in this case, as the probability distribution of the transmit rate scales with RSF whereas that of user's think time does not.

2) Open-loop traffic model: The file arrival process to $\mathrm{BS}_{j}$ from the network is now modeled as a Poisson process, i.e service rate and user's request rate are not correlated. The inter-arrival times between files which can be treated as a batch of packets corresponding to a particular packet session are assumed to be exponentially distributed with mean $\frac{1}{\lambda}$. The size of these bursts of packets (or files) is assumed to be exponentially distributed with mean $\frac{1}{\mu}$. Thus, the queue at $\mathrm{BS}_{j}$ can be modeled as a M/M/1 queue. The average service rate of this $\mathrm{M} / \mathrm{M} / 1$ queue for a RSF of $\alpha$ is $R(\alpha)$. The probability that $\mathrm{BS}_{j}$ is on (or has a non empty queue) can be given by,

$$
A_{f, j}=\mathbb{E}\left[\mathbf{1}_{j}\left(\alpha_{j}\right)\right]=\rho_{j}^{o}\left(\alpha_{j}\right)=\frac{\lambda}{\mu R_{j}\left(\alpha_{j}\right)}=\frac{\rho_{j}^{o}(1)}{\alpha_{j}} .
$$

The intensity $\rho^{o}\left(\alpha_{j}\right)$ can be interpreted as the activity factor of the interfering BS signifying the fraction of time it interferes with the video user. Thus, as RSF decreases activity factor increases, although $\alpha_{j}>\rho_{j}^{o}(1)$ for the queue to remain stable.

\footnotetext{
${ }^{2}$ It is assumed that the utilization of $\mathrm{BS}_{0}$ (and hence mean interference experienced by data users) does not vary with rate scaling by interfering BSs. This makes $h_{j}$ 's independent of RSF's.
} 


\section{Framework for Modeling Real-Time Video Packet Losses}

In this section, a framework is developed in order to highlight the dependence of packet losses on playback deadline violations and link throughput variations. To get insight into the improvement from interference shaping, the packet loss rate is analyzed based on the developed framework before benchmarking against perceptual QoE metrics.

Unlike data transmissions, live streaming and conversational services have strict delay constraints. In these services video frames are played in real-time as they are received. An increase in packet delay from its nominal value can lead to the packet missing its playback deadline, rendering it useless. We consider the streaming of a real-time video service where a startup delay of $\Delta t$ is allowed. Once the playback starts, no rebuffering is allowed. Startup delay in terms of frame period could be written as $\Delta t=a \Delta t_{F}$ where $a \in \mathbb{N}$ and $\Delta t_{F}$ is frame period ${ }^{3}$. Thus, for a smooth and continuous video playback, the video packets belonging to $(n+1)^{\text {th }}$ display slot (or $a$ frames) should be received at the video receiver within $\Delta t$ time of the start of the playback of $n^{\text {th }}$ display slot. It is assumed that the packets in the $n^{\text {th }}$ slot are independently decodable or at most depend on the previously decoded data. If $B_{n}$ denotes the bit rate of the coded video during the $n^{\text {th }}$ slot then the total bits that need to be delivered in this slot are $B_{n} \Delta t$ and if $P$ denotes the fixed size of video packets, then the number of packets required at decoder per slot interval of $\Delta t$ are given by $\left\lceil\frac{B_{n} \Delta t}{P}\right\rceil$. If $C_{n}$ denotes the capacity of the channel during the $n^{\text {th }}$ slot, then the fraction of packets lost during this slot is given by $\frac{\left(\left\lceil B_{n} \Delta t / P\right\rceil-\left\lceil C_{n} \Delta t / P\right\rceil\right)^{+}}{\left\lceil B_{n} \Delta t / P\right\rceil} \approx \frac{\left(B_{n}-C_{n}\right)^{+}}{B_{n}}$, where $(x)^{+}=\max (x, 0)$. The average packet loss rate (PLR) during the video transmission process can be written as,

$$
\operatorname{PLR}=\frac{1}{N} \sum_{n=1}^{N} \frac{\left(B_{n}-C_{n}\right)^{+}}{B_{n}},
$$

where $N$ corresponds to the length of video transmission in terms of display slots. For $N$ sufficiently large and assuming the channel statistics are stationary, the packet loss rate can be written as,

$$
\mathrm{PLR}=\mathbb{E}\left[\frac{\left(B_{n}-C_{n}\right)^{+}}{B_{n}}\right] .
$$

Obviously, a higher value of PLR would correspond to a lower video quality. As seen from (6), if the source encoding could track the channel rate there would be ideally no losses. However for real-time AVC video applications the adaptation would require changing the encoding parameters dynamically which would require exact channel state information at the encoder, which is usually not viable [23]. In some applications like broadcast/multicast this may never be possible. Losses would be higher if the channel rate experiences larger variability, which the slow adaptation would be unable to track.

Packet retransmissions are not considered as they would incur further delays. A similar information loss framework to capture losses due to bufferless streaming of variable bit rate video sources on constant bandwidth channel was introduced

\footnotetext{
${ }^{3}$ Typically in conversational services this delay and buffering is small [4].
}

in [24]. With the presented framework, the performance of any algorithm targeted to reduce the variability of wireless throughput and hence improve video quality in such delay constrained settings can be assessed by observing the improvement in the metric PLR defined in (6).

\section{INTERFERENCE SHAPING IN WIRELESS NETWORKS}

In this section, a straightforward approach for tackling the challenge of bursty interference described in previous sections is presented and analyzed.

Definition 3. Interference shaping: Temporal smoothing of the interference power through power/rate scaling of the interfering BSs' bursty best effort data.

Interference shaping can be triggered upon learning about the degradation of the nearby video user by the interfering BSs and on a particular set of subcarriers (for OFDMA systems). The maximum power scaling an interfering BS will be able to provide depends on its traffic load and delay constraints. In the ensuing sections we apply the above algorithm and also quantify the gains in some illustrative settings.

\section{A. Unicast Streaming}

We begin by evaluating the effect of interference shaping on unicast streaming. The video is assumed to be fed into the video buffer at the transmitter at constant rate. This assumption is reasonable and inconsequential to the performance analysis because: (i) video traffic could already be encoded as constant bit rate (CBR) video (ii) or, for variable bit rate video encoding, smoothing techniques already reduce the variability in the video rate. The same assumption was shown to cause only minimal additional play-back delay (order of milliseconds) at the user in [25]. To evaluate the effect of interference shaping on PLR we assume that the transmitted bit rate is equal to the mean channel capacity i.e. $B_{n}=\bar{C}(\vec{\alpha})$, where $\vec{\alpha}$ is the vector, whose $i^{\text {th }}$ element denotes the RSF of $i^{\text {th }}$ interfering BS. This is the case when the video encoding rate is determined by the mean channel bandwidth, where a receiver selects an appropriate stream that matches with the link capacity from the many streams encoded at different rates provided by video server. Then, the average fraction of packet losses as a function of the RSF can be written as

$$
\operatorname{PLR}(\vec{\alpha})=\mathbb{E}\left[\frac{\left(B_{n}-C_{n}\right)^{+}}{B_{n}}\right]=\frac{\mathbb{E}\left[\left(\bar{C}(\vec{\alpha})-C_{n}\right)^{+}\right]}{\bar{C}(\vec{\alpha})} .
$$

Based on the setup described in the previous sections, the mean channel capacity is

$\bar{C}(\vec{\alpha})=\mathbb{E}\left[\log \left(1+\frac{h_{0} \gamma}{1+\sum_{j=1}^{|J|}\left(h_{j}\left(\left(1+\gamma_{F j}\right)^{\alpha_{j}}-1\right)\right) \mathbf{1}_{j}}\right)\right]$,

where the expectation is over $\mathbf{1}_{j}$ for $j=1, \ldots, J$ and channel gains. The maximum rate that is allowed in $n^{\text {th }}$ slot is,

$$
C_{n}(\vec{\alpha})=\log \left(1+\frac{h_{0} \gamma}{1+\sum_{j=1}^{|J|}\left(h_{j}\left(\left(1+\gamma_{F j}\right)^{\alpha_{j}}-1\right)\right) \mathbf{1}_{j}}\right) .
$$




$$
\operatorname{PLR}\left(\alpha_{1}\right)=\frac{A_{f, 1}\left(1-A_{f, 1}\right) \log \left(\frac{1+h_{0} \gamma}{1+\frac{h_{0} \gamma}{1+\left(h _ { 1 } \left(\left(1+\gamma_{F 1}\right)^{\left.\left.\alpha_{1}-1\right)\right)}\right.\right.}}\right)}{A_{f, 1} \log \left(1+\frac{h_{0} \gamma}{1+\left(h _ { 1 } \left(\left(1+\gamma_{F 1}\right)^{\left.\left.\alpha_{1}-1\right)\right)}\right.\right.}\right)+\left(1-A_{f, 1}\right) \log \left(1+h_{0} \gamma\right)} .
$$

As an example, for the case with one dominant interfering base station $\left(\mathrm{BS}_{1}\right)$ to the video user and assuming fixed channel gains, PLR can be written as (11).

If the video user experiences interference from multiple independent bursty interferers, the burstiness and hence the PLR at $\alpha_{i}=1$ is expected to decrease. Hence, the gain in terms of PLR reduction and video quality improvement that can be obtained from interference shaping would be less as compared to the case with single dominant interferer. Fig. 2 shows the PLR reduction with five interferers having traffic intensities $\rho_{i}^{o}(1)=0.2,0.3,0.5,0.7$ and 0.8 for open loop traffic and traffic intensities $\rho_{i}^{c}(1)=1 / 3,1 / 2,1,2$ and 3 for closed loop traffic. The plot shows the variation of PLR for different channel gain vectors with $i^{\text {th }}$ element of the vector being the channel gain from $i^{\text {th }}$ interfering $\mathrm{BS}$ to the video user. Specifically $\vec{h}^{(1)}=[0.2,0.2,0.2,0.2,0.2], \vec{h}^{(2)}=$ $[0,0.2,0.2,0.2,0.4], \vec{h}^{(3)}=[0,0,0.2,0.2,0.6]$, and so on with $\vec{h}^{(5)}$ denoting the case with only one interferer. The average received SNR at both the video user and the best effort user is assumed to be same or $\gamma=\gamma_{F j}=10$, and $h_{0}$ is assumed to be unity. As shown in these plots, interference shaping decreases the packet loss rate monotonically to zero with $\mathrm{RSF}=1$ point denoting the baseline system with no interference shaping. However, the decrease in RSF would lead to an increase in file delays. This is investigated in detail in the next section. It is assumed that the variation in wireless capacity on the timescale of display slots is only due to interference (as channel gains are assumed to be fixed) and if this is not the case PLR would decrease with rate scaling but without going down to zero. However, as pointed out in [26] data rate variations in dense wireless networks are usually due to interference rather than short term channel fluctuations that can be compensated for by power control.

\section{B. Multicast Streaming}

Unicast streaming can be optimized for a particular pointto-point link. In a scenario where many users are watching the same video at a particular instant, unicast is inefficient and can put severe pressure on the limited resources. To cope with scenarios where a large number of users watch a live event or popular real-time video stream, multicast and broadcast streaming modes have been introduced to the broadband standards such as LTE and WiMAX [27].

Although efficient in network resource utilization, multicasting is difficult to adapt to wireless channels, which makes the reliable delivery of multimedia difficult. In dense wireless networks, multicast streaming adds additional relevance to the interference shaping approach. Often there may be correlation between the location of users in a particular multicast group, similarly some users would likely have common interfering

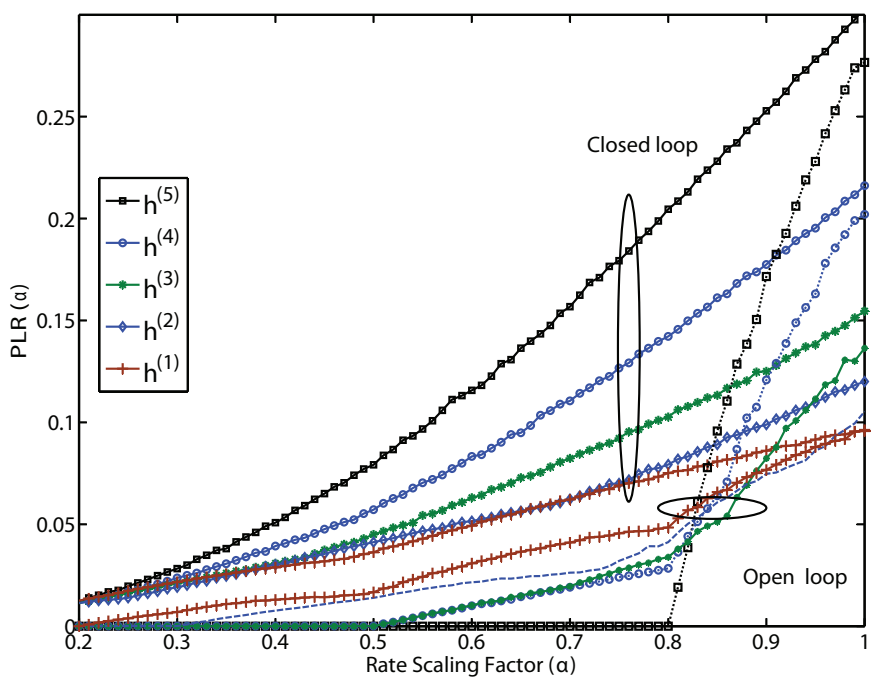

Fig. 2. Variation of packet loss rate of video user with RSF with multiple interferers

BSs. As an example, consider users in a stadium streaming live replays on their mobile devices with a nearby base station contributing bursty interference. If interference shaping is employed in such cases, then with the same modest losses for the single bursty data user, proportionally higher gains are obtained for the entire set of multicast video users.

This can be rigorously argued from a network wide sum QoE perspective. If $S_{\mathrm{QoE}}$ is the sum of QoE scores $\left(Q_{i}\right)$ across all users (set $U$, say) of which $U_{M}$ is the set of collocated multicast users sharing a common interferer $\mathrm{BS}_{k}$, with user $k$ consuming bursty data. Then,

$$
S_{\mathrm{QoE}}(1)=\sum_{i \in U} Q_{i}=\sum_{i \in U_{M}} Q_{i}+Q_{k}+\sum_{i \in U /\left\{U_{M}, k\right\}} Q_{i} .
$$

If the adoption of interference shaping by $\mathrm{BS}_{k}$ (by scaling rate with $\alpha$ ) leads to a corresponding increase of $\Delta Q_{i}$ in QoE scores for video users in $U_{M}$ and a corresponding $\Delta Q_{k}$ decrease for the best effort user of $\mathrm{BS}_{k}$. Then, the sum QoE at RSF of $\alpha$ is,

$$
\begin{aligned}
S_{\mathrm{QoE}}(\alpha) & =Q_{k}-\Delta Q_{k}+\sum_{i \in U_{M}}\left(Q_{i}+\Delta Q_{i}\right)+\sum_{i \in U /\left\{U_{M}, k\right\}} Q_{i} \\
& =S_{\mathrm{QoE}}(1)-\Delta Q_{k}+\sum_{i \in U_{M}} \Delta Q_{i}
\end{aligned}
$$

Thus, the gain in network wide sum QoE is proportional to the set of multicast users $\left|U_{M}\right|$. With $\left|U_{M}\right|=1$ the scenario becomes equivalent to unicast.

As mentioned earlier the defined metric of packet loss rate can only be a coarse measure of video quality because of the decoding interdependencies among the packets. Moreover PLR 

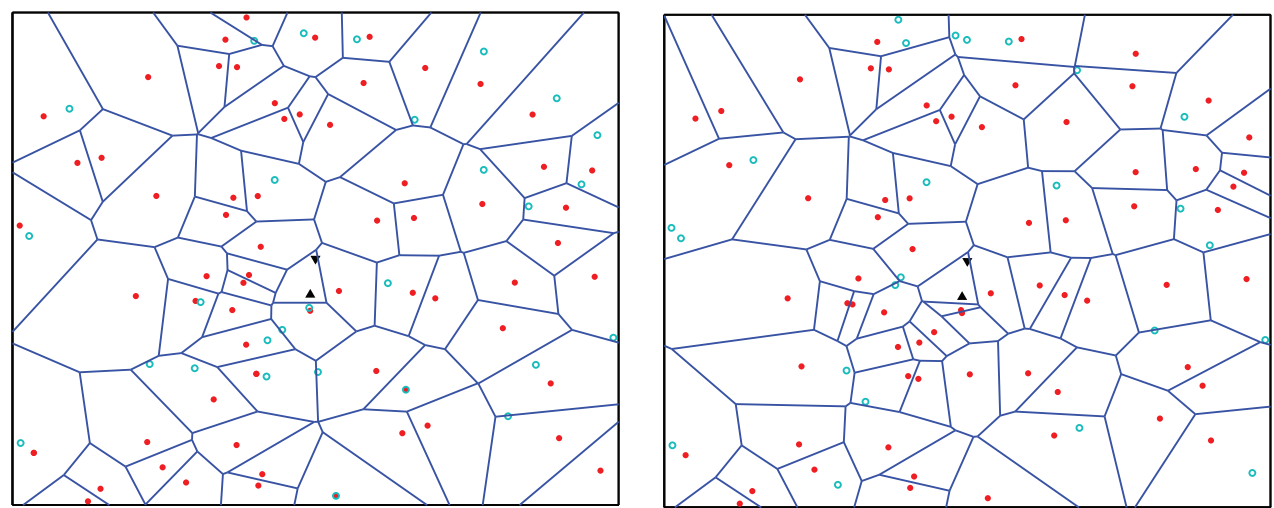

Fig. 3. The snapshots of changing locations of active BS for a particular realization of the 2D PPP describing the BS locations (the video UE is the inverted triangle, triangle symbol is the video BS, filled dots in the plane are active interfering BSs while hollow dots are the non active BSs in the plane).

is only a network level performance metric which cannot be directly correlated with end user QoE. The QoE assessment results presented in the Sec. IV will allow us to conclude with more confidence on the effect of interference shaping on perceived video quality.

\section{Mean capacity of video link with interference shaping}

Interference shaping reduces the packet loss rate and hence protects the video transmission from the distortions due to bursty interference. However, the quality of the original encoded video would depend on the encoding rate which is assumed to be matched to mean channel capacity in previous sections. With interference shaping, video user experiences reduced interference power but for a larger fraction of time due to increase in activity factor of interfering BSs. This makes the effect on the mean capacity non-intuitive. Hence, it becomes important to analyze the effect of the proposed algorithm on the mean channel capacity.

A cellular framework is used, with base stations randomly located according to a homogeneous Poisson point process (PPP), $\Phi$ where each BS transmits bursty traffic to their users. This extends the setting presented in [28] where every interfering base station was assumed to be active at all times. This model exploits the property that typical video lengths are much larger than the burst time scales of web traffic which are further larger than fast fading time scales. Thus, conditioning on the distance between the video UE and BS the set of active BSs may change while the run of the video stream as shown in Fig. 3. For a particular realization of the random BS locations, the video UE associates with the nearest BS. It is assumed that the other BSs in the network have the same activity factor, $A_{f}$. The distance of the video user to $\mathrm{BS}_{i}$ is given by $\mathrm{D}_{i}$. The path loss exponent is denoted by $\eta$ and the channels are assumed to have Rayleigh fading and hence exponential power gains denoted by $g_{i} \sim \exp (1)$. The transmit power of BS serving the video user is assumed to be $\gamma_{o}$ and that of other interfering BSs to be $\gamma_{1}$. The UE's distance from the serving BS is assumed to be $D$ and the fading gain to be $g$. Since current cellular networks are mostly interference limited, noise is ignored in the following analysis and interference is treated as noise. The $\mathrm{SIR}$ of the video user under the given system model is given by

$$
\mathrm{SIR}_{\mathrm{v}}=\frac{\gamma_{o} g D^{-\eta}}{I_{D}}
$$

where $I_{D}=\sum_{i \in \Phi_{a} / 0} \gamma_{1} g_{i} D_{i}^{-\eta}$ where $\Phi_{a}$ denotes the set of active base stations which is a subset of $\Phi$. It is assumed that a typical user achieves the Shannon bound for its spectral efficiency given by $\ln (1+$ SIR $)$ at its instantaneous SIR .

Lemma 1. Using the properties of PPP, the ergodic capacity of the video link (for the special case of $\eta=4$ ) is given by,

$$
\begin{aligned}
C_{v} & =\mathbb{E}\left[\log \left(1+\mathrm{SIR}_{\mathrm{v}}\right)\right]=\int_{t>0} \mathbb{P}\left(\log \left(1+\mathrm{SIR}_{\mathrm{v}}\right)>t\right) \mathrm{d} t \\
& =\int_{t>0} \frac{1}{1+A_{f} \kappa\left(\delta\left(2^{t}-1\right)\right)} \mathrm{d} t .
\end{aligned}
$$

where $\delta=\frac{\gamma_{1}}{\gamma_{o}}, \kappa(x)=\sqrt{x}\left(\frac{\pi}{2}-\arctan \left(\frac{1}{\sqrt{x}}\right)\right)$ and $A_{f}$ is the activity factor of interfering BSs.

Proof: See Appendix A.

The effect of an algorithm that changes the activity factor of interfering BSs $\left(A_{f}\right)$ or the transmit power (and hence $\delta$ ) can be accessed using the above expression. In this multi-cell system if upon learning the presence of a real-time video user and degradation of its QoE, the interfering BSs lower their transmit power by a PSF of $\beta$, i.e. $\gamma_{1}=\beta \gamma_{o}$. This leads to a decrease in their respective transmission rates, leading to an increase in their activity factor $\left(A_{f}\right)$. The exact relation between the power reduction and rate reduction is not tractable because of the coupling caused by interference between the BSs and hence such coupling is neglected in the following analysis. If $R_{o j}$ is the mean transmit rate of $\mathrm{BS}_{j}$ at $\beta=1$, then that at $\beta<1$ can be written as $\epsilon\left(R_{o j}, \beta\right) \beta R_{o j}$, where $\epsilon\left(R_{o j}, \beta\right)$ is a correction term which depends on $R_{o j}$ and $\beta$. It takes into account the effect of logarithmic relationship.

Therefore, the activity factor of interfering BSs at PSF $\beta$ is $A_{f}=\frac{\rho^{c}(1)}{\epsilon\left(R_{o j}, \beta\right) \beta+\rho^{c}(1)}$ for closed loop and $A_{f}=\frac{\rho^{o}(1)}{\epsilon\left(R_{o j}, \beta\right) \beta}$ for open loop. Using (15), for the closed loop traffic model, the ergodic capacity can be written as

$$
C_{v}=\int_{t>0} \frac{1}{1+\frac{\rho^{c}(1)}{\epsilon\left(R_{o j}, \beta\right) \beta+\rho^{c}(1)} \kappa\left(\beta\left(2^{t}-1\right)\right)} \mathrm{d} t .
$$




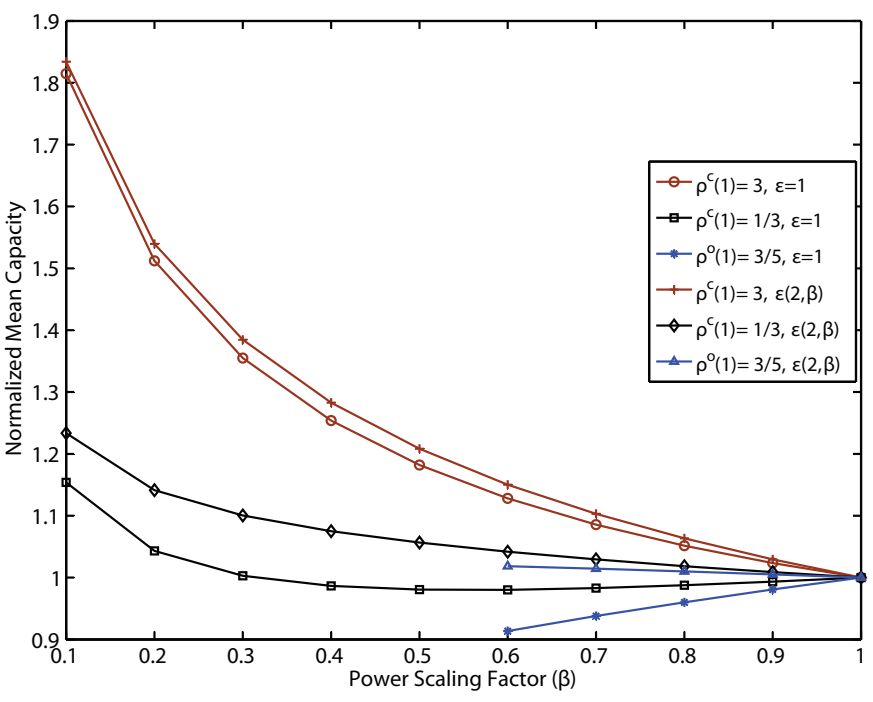

Fig. 4. Variation of the mean capacity of a typical video user as a fraction of the original capacity (without interference shaping) as PSF is varied.

Neglecting the correction term (keeping it as unity) will give a lower bound on mean capacity. Fig. 4 shows the variation of the mean capacity with and without incorporating correction term for both open loop and closed loop versus the change in the PSF. The original rate value used in correction term is 2 bits/s/Hz. The mean capacity increases for both types of traffic models at the given rate. Also, as seen with the lower bound, for the open loop model the mean capacity can decrease no less than a factor of 0.91 and with closed loop traffic, mean capacity would increase for all rate values. The capacity variation trend for the scenario where interferers consist of both kind of BSs could be a subject of future investigation.

\section{INTERFERENCE SHAPING AND QUALITY OF EXPERIENCE}

As observed in the previous section, interference shaping reduces the packet loss rate and throughput variation by decreasing the peak power (and hence rate) of interfering bursty transmitter. Hence, it may lead to QoE degradation for bursty non video traffic users in exchange for improving the QoE of real-time video users. This section aims the quantify this tradeoff.

\section{A. Real-time video users}

The following approach is used to assess the subjective user experience. The transmission of the encoded real-time video streaming service is simulated. The distortions comprise both the encoding and the packet losses governed by the earlier presented packet loss model. An objective full reference quality metric multi-scale structural similarity index (MSSSIM) [15] is used for the frame level quality assessment of the decoded distorted video stream. In order to predict the overall objective quality score, the frame-level quality scores are temporally pooled accounting for the fact that users respond strongly to drops in video quality [8]. Unlike temporal averaging, this pooling strategy takes into account the effect of bad quality experienced in the recent past into the objective estimate of quality at the current instant. The overall objective score thus obtained is referred to as hysteresis based MS-SSIM (or H-MS-SSIM). These are then mapped to Differential Mean Opinion Scores (DMOS) (ranging from 1-100) according to [29] which are a measure of the QoE, as higher the DMOS lower the user satisfaction with the quality.

Definition 4. The QoE improvement for the video user with respect to rate scaling factor, $\vec{\alpha}$, is defined as

$$
\operatorname{DMOS}(\mathrm{RSF}=1) / \operatorname{DMOS}(\operatorname{RSF}=\vec{\alpha}) .
$$

1) Video Quality Assessment: Various full reference metrics are available for assessing the quality of video. PSNR (peak signal to noise ratio) which is inversely proportional to the mean squared error between the reference and distorted video, is popular but has low correlation with actual perceptual quality [30]. PSNR can give the same values for many different types of distortion like source compression and packet loss, whereas the perceptual effect of these is often drastically different. In [15], a full reference metric MS-SSIM was proposed that has been found to predict the perceptual quality with very high correlation. It is an improvement over the single scale structural similarity index which exploited the fact that there is a definite "structure" in natural images and the change in structure information would be proportional to quality degradation. The SS-SSIM can be calculated as

$$
\operatorname{SSIM}(\mathrm{x}, \mathrm{y})=\frac{\left(2 \mu_{x} \mu_{y}+C_{1}\right)\left(2 \sigma_{x y}+C_{2}\right)}{\left(\mu_{x}^{2}+\mu_{y}^{2}+C_{1}\right)\left(\sigma_{x}^{2}+\sigma_{y}^{2}+C_{2}\right)},
$$

where $x$ and $y$ are pristine and distorted image signals respectively, $\mu_{x}, \mu_{y}, \sigma_{x}, \sigma_{y}$ and $\sigma_{x y}$ are the mean intensity of $x$, mean intensity of $y$, variance of $x$, variance of $y$ and covariance of $x$ and $y$ respectively and $C_{1}$ and $C_{2}$ are stabilizing constants.

The source video "Pedestrian Area" in RAW uncompressed progressive scan YUV420 format with a resolution of $768 \times 432$ and frame rate of 30 frames per second (f/s) from LIVE video database [31][32] is used. The video chosen depicted the scene of a busy market place with people moving in and out of frame with camera remaining still. The JM reference software (Version 13.1) [33] available from the Joint Video Team (JVT) was used for H.264/AVC encoding with base-line profile with no $\mathrm{B}$ frames was used. The reference video was encoded with bit rate of $1 \mathrm{Mbps}^{4}$ and with three slice groups. The other parameters of encoding used were as specified in [30]. Since we aimed for conversational and realtime video services, the packet sizes were kept between 100 and 300 bytes as recommended in [4].

2) Quality of Experience Assessment Results: The scenario with a single dominant interfering BS is first simulated. It is assumed that the average received SNR at the video user and best effort users are the same, $\gamma=\gamma_{F 1}=10$. Each $h_{i}$ is assumed to be constant across slots and equal to unity and $\rho^{o}(1)=0.5$. Variation in per frame value of MS-SSIM for two values of rate scaling factors is shown in Fig. 5. As seen the

\footnotetext{
${ }^{4}$ Mean capacity was assumed to be fixed with interference shaping and sufficient to support the video bit rate. This was to focus on the QoE improvement provided by the decrease in throughput variation only and not that by the increase in the mean capacity (as it is scenario dependent).
} 


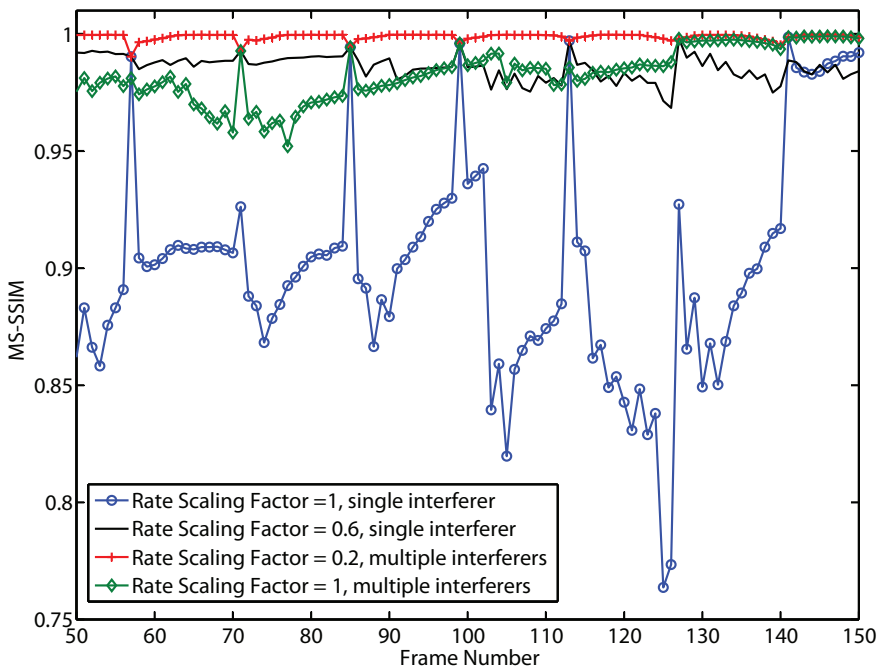

Fig. 5. Sample path in time of per frame MS-SSIM for the pedestrian sequence with interference shaping in presence of a single dominant interferer and multiple interferers.

quality improvement is uniform throughout the video sequence and the average quality is not dominated by a window of high quality sequence. Also the temporal variation in quality is considerably reduced with the shaping of interference. Fig. 6 shows the variation of the overall objective quality score H-MS-SSIM and the corresponding QoE (measured as the inverse of DMOS) as RSF is varied for the video sequence. As seen from the plot, the proposed interference shaping algorithm improves the QoE of the video user monotonically with decreasing RSF. In particular, at an RSF value of 0.6 and 0.55 the $\mathrm{QoE}$ is $2 \mathrm{x}$ and $\sim 3 \mathrm{x}$ respectively with respect to the original value $(\mathrm{RSF}=1)$.

Fig. 5 also shows the temporal variation in the objective scores for the same video sequence with five interfering BSs having traffic intensities $\rho_{i}(1)=0.2,0.3,0.5,0.7$ and 0.8 , channel gain from interferers $\vec{h}=\vec{h}^{(1)}$ and $h_{0}$ are 1. Fig. 6 shows the variation in H-MS-SSIM and perceptual quality for this case. The variation is shown with the RSF of the base station with minimum traffic intensity. In simulations, the interfering BSs scale simultaneously and up to their delay constraint which is assumed to be such that for $j^{\text {th }} \mathrm{BS}$ $\alpha_{j} \geq \rho_{j}^{o}(1)+0.05$. Again the QoE increases monotonically with decreasing RSF and the $\mathrm{QoE}$ is $\sim 1.5 \mathrm{x}$ at $\mathrm{RSF}=0.6$ with respect to the original value $(\mathrm{RSF}=1)$. Thus, the improvement seen in single interferer case carries over to multiple interferers, too. Table I shows the effect of interference shaping on the time averaged quality scores and coefficient of variation for quality (CoVQ) defined as $\frac{\sqrt{\operatorname{Var}[Q]}}{\mathbb{E}[Q]}$ where $Q$ is the frame level objective score. Considerable improvement can be observed in temporal fluctuations of quality with interference shaping. As noted earlier, user experience is maximized by maximizing the average frame quality and minimizing the variations in quality. Thus, across various metrics considered, the proposed approach leads to monotonic improvement in QoE of video users.

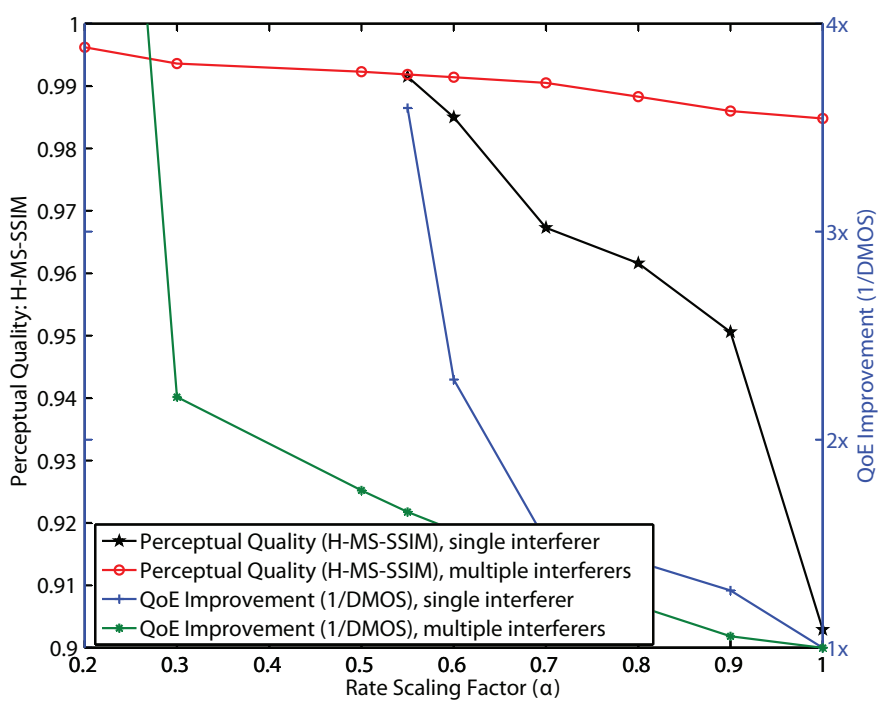

Fig. 6. Variation of overall quality (H-MS-SSIM) and QoE for the pedestrian sequence with interference shaping in presence of single and multiple interferers.

TABLE I

COMPARISON OF COQV AND AVERAGE FRAME BASED MS-SSIM

\begin{tabular}{|l|c|c|c|c|}
\hline \multirow{2}{*}{} & \multicolumn{2}{|c|}{$\begin{array}{c}\text { Single } \\
\text { Interferer }\end{array}$} & \multicolumn{2}{c|}{$\begin{array}{c}\text { Multiple } \\
\text { Interferers }\end{array}$} \\
\cline { 2 - 5 } & CoQV & MS-SSIM & CoQV & MS-SSIM \\
\hline Without interference shaping & 0.0694 & 0.9099 & 0.0119 & 0.9851 \\
With interference shaping & 0.0097 & 0.9859 & 0.0076 & 0.9965 \\
\hline
\end{tabular}

\section{B. Best-effort traffic users}

Web browsing and file downloads are considered as the typical best effort applications in the following evaluation. Rate backoff by the interfering BS due to scaling of transmit power would decrease the file download speed or increase the mean page response time for its users. The mean web page response time (or file download time) can be expressed as

$$
\mathbb{E}[D]=D_{\text {backbone }}+\frac{1}{\mu R(\alpha)},
$$

where $D_{\text {backbone }}$ accounts for the latency due to backbone delay and web server response time, and $\frac{1}{\mu R(\alpha)}$ is the wireless link delay for the average page (or file) size of $\frac{1}{\mu}$. The backbone delay is neglected in the following analysis but it is worth noting that since the average mean response time increases with decreasing RSF, $\alpha$, the fractional increase may depend on the relative values of the backbone and access link delays.

The user experience of web based file downloads was shown to exhibit strong logarithmic dependence on the bandwidth of the network in [16]. The basis of this relation is Weber-Fechner law that establishes the logarithmic nature of human sensitivity to any stimulus. The exact logarithmic nature may depend on the context. In particular for file downloads of size $2.5 \mathrm{MB}$,

TABLE II

MEAN OPINION SCORES

\begin{tabular}{|c|c|c|c|c|c|}
\hline MOS & 5 & 4 & 3 & 2 & 1 \\
\hline Quality & Excellent & Good & Fair & Poor & Bad \\
\hline
\end{tabular}


the QoE (measured as MOS defined in Table II) as a function of bandwidth is given as,

$$
\mathrm{QoE}=0.775 \log (\mathrm{BW})+1.268,
$$

where $\mathrm{BW}$ is the normalized bandwidth. With rate scaling the above relation can be written as

$$
\mathrm{QoE}=0.775 \log (\alpha)+1.268 .
$$

For web browsing applications, a similar logarithmic dependency of QoE on session time (page download time) was shown to be highly accurate in [17]. The following relation accounting for the QoE of a user transitioning from a fast to slow network (which is the case with interference shaping) is used.

$$
\text { QoE }=5.57-1.64 \log (\text { pageloadtime }) \text {. }
$$

With rate scaling, the above relation becomes,

$$
\mathrm{QoE}=5.57-1.64 \log \left(\frac{1}{\mu R(\alpha)}\right) \text {. }
$$

In the scenario where the initial $(\mathrm{RSF}=1)$ downlink bandwidth is $2048 \mathrm{Kbits} / \mathrm{s}$, the QoE variation with RSF for file download service is shown in Fig. 7. In the same plot, the QoE degradation for a web surfing user is shown for the case where initial session time is 1.4 secs. As shown, for RSF of up to 0.6 the MOS scores for both the use cases are quite close to 4 which is considered a good user satisfaction level.

Fig. 8 demonstrates the evident trade-off between the QoE of data users and that of real-time video users under the proposed interference shaping. Aggressive rate scaling by interfering BSs improves the QoE of video user by 2-3x while reducing the QoE of best effort users by a factor of $0.7 \mathrm{x}$ (from $1.35 \mathrm{x}$ to $1 \mathrm{x}$ ), or from excellent (MOS $>4$ ) to good (MOS $>3)$. As noted earlier, often in resource allocation algorithms preferential treatment is given to video traffic as compared to best effort traffic by assigning the highest QoS class to the former. So, in a way the proposed approach can be treated as a case of preferential treatment across the base stations.

\section{IMPLEMENTATION DETAILS OF INTERFERENCE SHAPING}

The proposed algorithm can be implemented in current standards like LTE without much incremental effort. The key features required for the implementation of the presented algorithm are described below.

- QoE monitoring and feedback : Interference shaping requires knowledge about the QoE of video user(s) to be available with the network. The QoE can be monitored and evaluated according to the presented QoE model on the client/user. Then, the existing feedback channels may be used for the calculated QoE score or dedicated uplink data channels may be used. The monitoring and feedback of QoE metrics is an active area in 3GPP standards [34], [35]. 3GPP DASH specification [36] defines the QoE measurement and reporting capabilities for clients. So this feature is well within the scope.

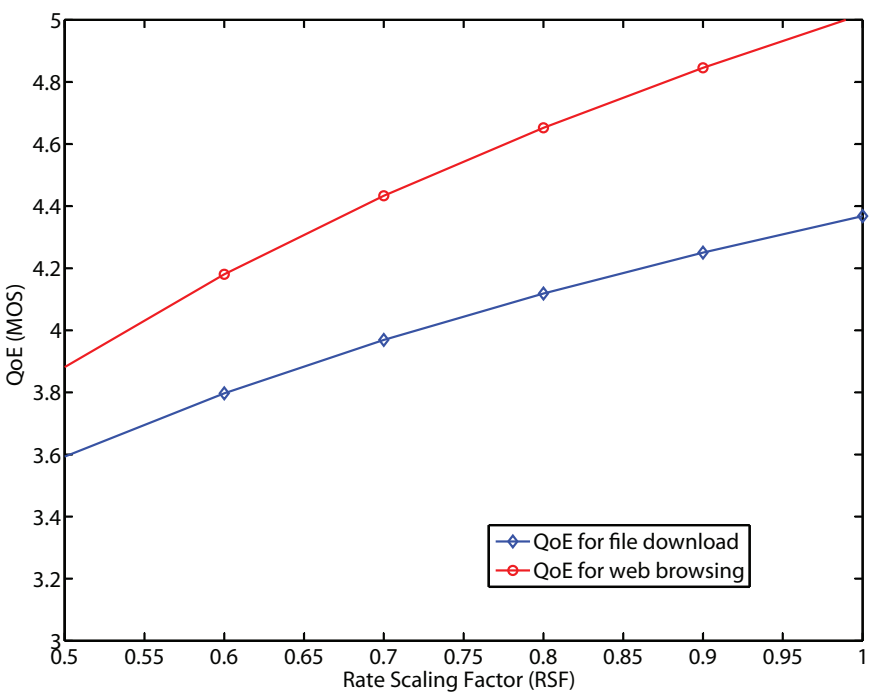

Fig. 7. QoE variation for best effort users with rate scaling

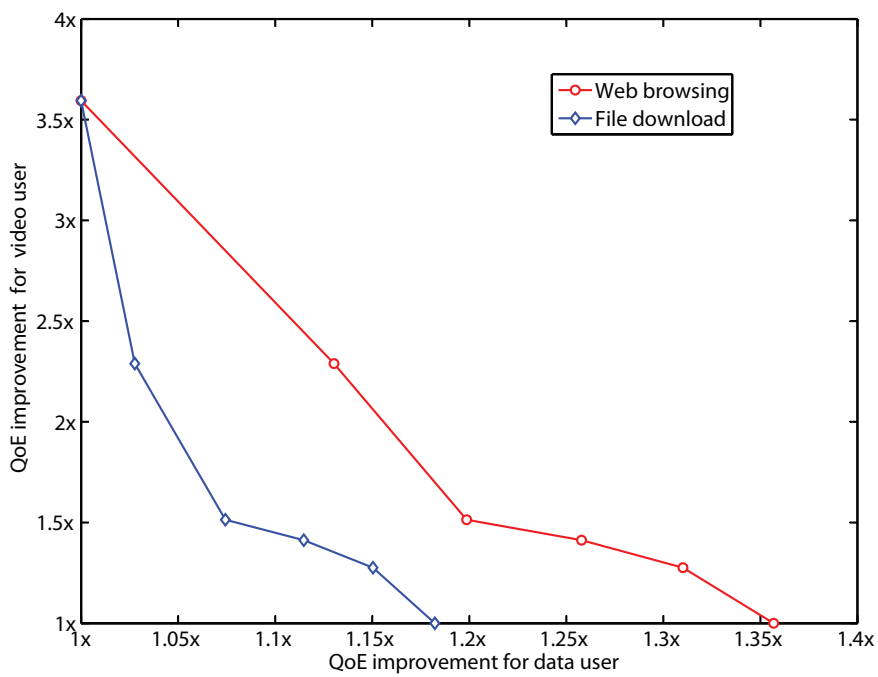

Fig. 8. Trade-off curve between QoE improvement of data user and that of video user.

- QoE information sharing: Once the QoE status is known to the network, then the corresponding information can be shared among the base stations either through the backhaul or over dedicated overhead channels. In fact, information sharing among nearby base stations is already prevalent in LTE systems. Techniques like coordinated multipoint (CoMP) transmission [37] involves comparatively a much larger amount of side information sharing for a much lower gain.

- QoE aware power scaling: The radio resource management (RRM) engine present at every base station is responsible for allocating resources in terms of bandwidth, power and time for each user. This engine is made aware of the type of traffic for each user through QoS class indicators (QCI) and QoS classes (specified in [38]) by the network. As an example, real-time video traffic would have a QCI value in the range of 1-3 whereas that for the best effort data would be in the range of 7-9 [39]. The resource allocation is then done in accordance to QoS 
requirements. Thus, the required power scaling can be handled similarly through a possible QoE specifier made available to RRM by the network.

Interference shaping can also be applicable in scenarios where a BS transmits a mix of real-time video and busrty data. For illustration assume $\mathrm{BS}_{i}$ and $\mathrm{BS}_{j}$ carry a mix of realtime video and bursty data over an OFDMA cellular system. Let us further assume $\mathrm{BS}_{i}$ uses frequency band $\mathrm{F}_{A}$ for realtime video and $\mathrm{F}_{B}$ for data. If $\mathrm{BS}_{j}$ 's video user is allocated frequency band $\mathrm{F}_{B}$ and reports $\mathrm{QoE}$ degradation, $\mathrm{BS}_{i}$ can scale the power allocated to subband $\mathrm{F}_{B}$ and shape the interference. Similarly, this can be extended to those $\left(\mathrm{F}_{A}, \mathrm{~F}_{B}\right)$ combinations in which data user is a co-channel interferer to video user ${ }^{5}$.

\section{CONCLUSION}

The burstiness of wireless links can lead to severe degradation of perceptual quality and hence the Quality of Experience (QoE) of real-time video users. Packet loss rate and throughput variations decrease with interference shaping across various scenarios. Through the cellular framework it was shown that, perhaps surprisingly, mean capacity did not decrease much with interference shaping. The developed model could also be useful in assessing video specific algorithms in similar settings. The presented work integrates subjective effects of video users such as hysteresis and quality variation into the performance analysis, and to the best of our knowledge, is the first work to do so. Use of recently developed web QoE models lead to a stronger conclusion on the affect of interference shaping on best-effort users. With interference shaping, mean quality of the received video increases and quality variation decreases leading to a significant improvement in the QoE of video users with a slight decrease in the QoE of best-effort users. The presented algorithm introduces a trade-off between the QoE of video users and web users and an optimal balance depends on the context. Another takeaway from this work is that there is considerable potential for improving QoE of users in the network by traffic shaping across wireless access points or BSs. Future work could involve further exploration along these dimensions by including QoE awareness in interference management techniques like Fractional Frequency Reuse (FFR) and Coordinated Multi-Point (CoMP) and in offloading and load balancing techniques.

\section{ACKNOWLEDGMENT}

The authors appreciate the insights shared by Anush Moorthy, Michele Saad, and Dr. Alan Bovik on video quality assessment metrics and their mapping to differential mean opinion scores (DMOS).

\section{APPENDIX A}

\section{DISTRIBUTION OF VIDEO USER SIR IN PRESENCE OF BURSTY INTERFERERS}

Proof: The density of the PPP $(\Phi)$ denoting the location of the BSs is assumed to be $\nu$. Herein it is assumed that the BSs

\footnotetext{
${ }^{5}$ In combinations where video users' transmission interfere with each other there is no advantage of shaping interference as the interference would not be bursty.
}

transmit independently with equal activity factors $\left(\operatorname{Pr}\left(\mathbf{1}_{n}=\right.\right.$ $\left.1)=A_{f}\right)$. Thus, the average density of the interfering base stations is $\nu A_{f}=\nu_{1}$ (say). The distribution of the SIR is given by

$$
\begin{aligned}
\mathbb{P}\left(\mathrm{SIR}_{\mathrm{v}}>\mathbb{A}\right) & =\mathbb{P}\left(g>\frac{\mathbb{A} D^{\eta} I_{D}}{\gamma_{o}}\right)=\mathbb{E}\left[\exp \left(-\frac{\mathbb{A} D^{\eta} I_{D}}{\gamma_{o}}\right)\right] \\
& =\mathcal{L}_{I_{D}}(s),
\end{aligned}
$$

where $\mathcal{L}_{I_{D}}(s)$ denotes the Laplace transform of interference with $s=-\frac{\mathbb{A} D^{\eta}}{\gamma_{0}}$. The density of the interfering BSs is the original density thinned by probability of transmission i.e. $\nu_{1}$ (in [28] it was assumed that all BSs are active and the set of interfering BS had the same density as the set of all BSs). Thus, the Theorem 2 of [28] can be used with the thinned density to obtain with Laplace transform of the interference as (assuming $\eta=4$ )

$$
\mathcal{L}_{I_{D}}(s)=\exp \left(-\pi D^{2} \nu_{1} \kappa\left(\frac{\gamma_{1}}{\gamma_{o}} \mathbb{A}\right)\right),
$$

where $\kappa(x)=\sqrt{x}\left(\frac{\pi}{2}-\arctan \left(\frac{1}{\sqrt{x}}\right)\right)$. The distance of the video user to the nearest BS i.e $D$ is random and its distribution from can be given by

$$
f_{D}(d)=e^{-\lambda \pi d^{2}} 2 \pi \lambda d
$$

Therefore,

$$
\begin{aligned}
\mathbb{P}\left(\mathrm{SIR}_{\vee}>\mathbb{A}\right) & =\int_{0}^{\infty} \exp \left(-\pi D^{2} \nu_{1} \kappa\left(\frac{\gamma_{1}}{\gamma_{o}} \mathbb{A}\right)\right) \exp \left(-\nu \pi d^{2}\right) 2 \pi \nu d \mathrm{~d} d \\
& =\int_{0}^{\infty} \exp \left(-\left(\frac{\nu_{1}}{\nu} \kappa\left(\frac{\gamma_{1}}{\gamma_{o}} \mathbb{A}\right)\right) x\right) \mathrm{d} x \\
& =\frac{1}{1+A_{f} \rho(\kappa \mathbb{A})},
\end{aligned}
$$

where $\delta=\frac{\gamma_{1}}{\gamma_{o}}$ and $\kappa(x)=\sqrt{x}\left(\frac{\pi}{2}-\arctan \left(\frac{1}{\sqrt{x}}\right)\right)$. The probability of coverage expression derived above gives the complementary cumulative distribution function (CCDF) of SIR at the video user.

\section{REFERENCES}

[1] Cisco, "Cisco Visual Networking Index: Global Mobile Data Traffic Forecast Update, 2011-2016." [Online]. Available : http://goo.gl/xxLT.

[2] Nokia, "Quality of experience (QoE) of mobile services: Can it be measured and improved?." [Online]. Available : http://goo.gl/mpyjM.

[3] ITU- International Telecommunication Union, "Definition of quality of experience (QoE)," Reference: TD 109rev2 (PLEN/12), Jan. 2007.

[4] T. Stockhammer, M. Hannuksela, and T. Wiegand, "H.264/AVC in wireless environments," IEEE Trans. Circuits Syst. Video Technol., vol. 13, pp. 657-673, July 2003.

[5] M. Claypool and J. Tanner, "The effects of jitter on the peceptual quality of video," in Proceedings of ACM international conference on Multimedia, pp. 115-118, ACM, Nov. 1999.

[6] S. Gulliver and G. Ghinea, "The perceptual and attentive impact of delay and jitter in multimedia delivery," IEEE Trans. Broadcast., vol. 53, pp. 449-458, June 2007.

[7] T. Lakshman, A. Ortega, and A. Reibman, "VBR video: tradeoffs and potentials," Proceedings of the IEEE, vol. 86, pp. 952-973, May 1998.

[8] K. Seshadrinathan and A. Bovik, "Temporal hysteresis model of time varying subjective video quality," in IEEE international conference on acoustics, speech and signal processing (ICASSP), pp. 1153-1156, May 2011.

[9] Ortiva Wireless, "Optimizing Mobile Video." [Online]. Available: http: //www.ortivawireless.com/p/WPOptimizingMobileVideo.pdf.

[10] A. Damnjanovic, J. Montojo, Y. Wei, T. Ji, T. Luo, M. Vajapeyam, T. Yoo, O. Song, and D. Malladi, "A survey on 3GPP heterogeneous networks," IEEE Wireless Commun. Mag., vol. 18, p. 10, June 2011. 
[11] S. Thakolsri, W. Kellerer, and E. Steinbach, "QoE-based cross-layer optimization of wireless video with unperceivable temporal video quality fluctuation," in "Proc. of IEEE International Conference on Communications", pp. 1-6, June 2011.

[12] V. Joseph and G. de Veciana, "Variability aware network utility maximization," CoRR, vol. abs/1111.3728, 2011.

[13] K. Piamrat, K. D. Singh, A. Ksentini, C. Viho, and J.-M. Bonnin, "QoE-Aware scheduling for video-streaming in High Speed Downlink Packet Access," in Wireless Communications and Networking Conference (WCNC), 2010 IEEE, pp. 1-6, Apr. 2010.

[14] S. Thakolsri, S. Khan, E. Steinbach, and W. Kellerer, "QoE-driven crosslayer optimization for High Speed Downlink Packet Access," Journal of Communications, vol. 4, no. 9, 2009.

[15] Z. Wang, E. Simoncelli, and A. Bovik, "Multiscale structural similarity for image quality assessment," in Proc. of the Asilomar Conference on Signals, Systems and Computers, vol. 2, pp. 1398-1402, Nov. 2003.

[16] P. Reichl, S. Egger, R. Schatz, and A. D'Alconzo, "The logarithmic nature of QoE and the role of the Weber-Fechner law in QoE assessment," in Proc. of IEEE International Conference on Communications, pp. 1-5, May 2010.

[17] E. Ibarrola, F. Liberal, I. Taboada, and R. Ortega, "Web QoE evaluation in multi-agent networks: validation of ITU-T G.1030," in Proc. of International Conference on Autonomic and Autonomous Systems, ICAS, pp. 289-294, Apr. 2009.

[18] C. Shannon, "Communication in the presence of noise," Proceedings of the IRE, vol. 37, pp. 10 - 21, Jan. 1949.

[19] 3GPP, "Evolved Universal Terrestrial Radio Access (E-UTRA); Physical layer procedures (Release 10)," TR 36.213, 2011.

[20] A. Wyner, "Shannon-theoretic approach to a Gaussian cellular multipleaccess channel," IEEE Trans. Inf. Theory, vol. 40, pp. 1713 -1727, Nov. 1994.

[21] J. Xu, J. Zhang, and J. Andrews, "On the accuracy of the Wyner model in cellular networks," IEEE Trans. Wireless Commun., vol. 10, pp. 30983109, Sept. 2011.

[22] L. Kleinrock, Queueing Systems, vol. 2. John Wiley \& Sons, 1975.

[23] T. Stockhammer and M. Hannuksela, "H.264/AVC video for wireless transmission," IEEE Trans. Wireless Commun., vol. 12, pp. 6-13, Aug. 2005.

[24] M. Reisslein and K. Ross, "Call admission for prerecorded sources with packet loss," IEEE J. Sel. Areas Commun., vol. 15, pp. 1167-1180, Aug. 1997.

[25] S. Deb, S. Jaiswal, and K. Nagaraj, "Real-time video multicast in WiMAX networks," in Proc. of IEEE INFOCOM, pp. 1579-1587, Apr. 2008.

[26] V. Chandrasekhar, J. Andrews, and A. Gatherer, "Femtocell networks: a survey," IEEE Commun. Mag., vol. 46, pp. 59 -67, Sept. 2008.

[27] K. Etemad and L. Wang, "Multicast and broadcast multimedia services in mobile WiMAX networks," IEEE Commun. Mag., vol. 47, pp. 84-91, Oct. 2009.

[28] J. Andrews, F. Baccelli, and R. Ganti, "A tractable approach to coverage and rate in cellular networks," IEEE Trans. Commun., vol. 59, pp. 31223134, Nov. 2011.

[29] H. Sheikh, M. Sabir, and A. Bovik, "A statistical evaluation of recent full reference image quality assessment algorithms," IEEE Trans. Image Process., vol. 15, pp. 3440-3451, Nov. 2006.

[30] A. Moorthy, K. Seshadrinathan, R. Soundararajan, and A. Bovik, "Wireless video quality assessment: a study of subjective scores and objective algorithms," IEEE Trans. Circuits Syst. Video Technol., vol. 20, pp. 587 599, Apr. 2010.

[31] K. Seshadrinathan, R. Soundararajan, A. Bovik, and L. Cormack, "Study of subjective and objective quality assessment of video," IEEE Trans. Image Process., vol. 19, pp. 1427-1441, June 2010.

[32] K. Seshadrinathan, R. Soundararajan, A. Bovik, and L. Cormack, "A subjective study to evaluate video quality assessment algorithms," in SPIE Proceedings of Human Vision and Electronic Imaging, Jan. 2010.

[33] Joint Video Team, "H.264/mpeg-4 AVC Reference Software Manual." [Online]. Available: http://iphome.hhi.de/suehring/tml/JM(JVT-X072) .pdf.

[34] Telefon AB LM Ericsson, ST-Ericsson SA, "QoE for HTTP Streaming," Tdoc S4-AHI118. Online available at: http://www.3gpp.org/ftp/tsg_sa/WG4_CODEC/Adhoc_MBS/Docs_AHI/S4-AHI118.zip.

[35] Huawei Technologies Co. Ltd., "Impact of Rebuffering on QoE," Tdoc S4-110404. Online available at: http://ftp.3gpp.org/ftp/tsg_sa/WG4_CODEC/TSGS4_64/docs/S4110404.zip.
[36] 3GPP, "Transparent end-to-end packet switched streaming service (PSS); Progressive download and dynamic adaptive streaming over HTTP (3GP-DASH), Release 10," TS 26.247 v 10.1.0, June 2011.

[37] 3GPP, "Coordinated multi-point operation for LTE physical layer aspects, Release 11," TR 36.819 v 11.1.0.

[38] 3GPP, "Quality of Service (QoS) concept and architecture, Release 10," TS 23.107 v 10.2.0.

[39] 3GPP, "General Packet Radio Service (GPRS) enhancements for Evolved Universal Terrestrial Radio Access Network (E-UTRAN) access (Release 8)," TS 23.401 v 8.1.0, 2008.

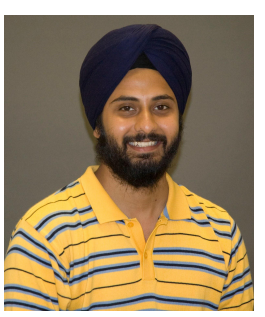

Sarabjot Singh (S'09) received his B.Tech degree in Electronics and Communication Engineering from IIT Guwahati, India, in 2010 and was awarded the President of India Gold Medal. He is currently working towards his Ph.D. at UT Austin where his research is focused on the QoE-aware design and analysis of heterogeneous wireless systems using tools from stochastic geometry, optimization, communication theory, and behavioral science. He is also interested in interference modeling and management in wireless systems, radio resource management for OFDMA systems, and belief propagation techniques for cellular systems. $\mathrm{He}$ has held internship positions at Intel Corporation, Santa Clara, CA, Qualcomm Inc, San Diego, CA and Indian Institute of Science, Bangalore.

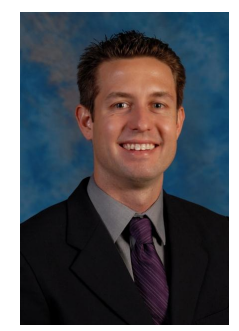

Jeffrey Andrews (S'98, M'02, SM'06) received the B.S. in Engineering with High Distinction from Harvey Mudd College in 1995, and the M.S. and Ph.D. in Electrical Engineering from Stanford University in 1999 and 2002, respectively. He is a Professor in the Department of Electrical and Computer Engineering at the University of Texas at Austin, where he was the Director of the Wireless Networking and Communications Group (WNCG) from 2008-12. He developed Code Division Multiple Access systems at Qualcomm from 1995-97, and has consulted for entities including the WiMAX Forum, Microsoft, Apple, Clearwire, Palm, Sprint, ADC, and NASA.

Dr. Andrews is co-author of two books, Fundamentals of WiMAX (PrenticeHall, 2007) and Fundamentals of LTE (Prentice-Hall, 2010), and holds the Earl and Margaret Brasfield Endowed Fellowship in Engineering at UT Austin, where he received the ECE department's first annual High Gain award for excellence in research. He is a Senior Member of the IEEE, a Distinguished Lecturer for the IEEE Vehicular Technology Society, served as an associate editor for the IEEE Transactions on Wireless Communications from 2004-08, was the Chair of the 2010 IEEE Communication Theory Workshop, and is the Technical Program co-Chair of ICC 2012 (Comm. Theory Symposium) and Globecom 2014. He has also been a guest editor for two recent IEEE JSAC special issues on stochastic geometry and femtocell networks.

Dr. Andrews received the National Science Foundation CAREER award in 2007 and has been co-author of five best paper award recipients, two at Globecom (2006 and 2009), Asilomar (2008), the 2010 IEEE Communications Society Best Tutorial Paper Award, and the 2011 Communications Society Heinrich Hertz Prize. His research interests are in communication theory, information theory, and stochastic geometry applied to wireless cellular and ad hoc networks. 


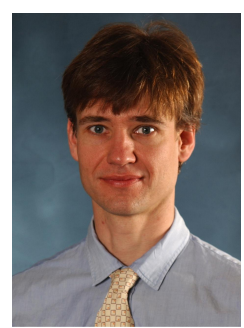

Gustavo de Veciana (S'88-M'94-SM'01-F'09) received his B.S., M.S, and Ph.D. in electrical engineering from the University of California at Berkeley in 1987,1990 , and 1993 respectively. He is currently a Professor at the Department of Electrical and Computer Engineering and recipient of the Temple Foundation Centennial Fellowship. He served as the Director and Associate Director of the Wireless Networking and Communications Group (WNCG) at the University of Texas at Austin, from 2003-2007. His research focuses on the analysis and design of wireless and wireline telecommunication networks; architectures and protocols to support sensing and pervasive computing; applied probability and queueing theory. Dr. de Veciana has served as editor for the IEEE/ACM Transactions on Networking. He was the recipient of a National Science Foundation CAREER Award 1996, co-recipient of the IEEE William McCalla Best ICCAD Paper Award for 2000, co-recipient of the Best Paper in ACM Transactions on Design Automation of Electronic Systems, Jan 2002-2004, co-recipient of the Best Paper in the International Teletraffic Congress (ITC-22) 2010, and of the Best Paper in ACM International Conference on Modeling, Analysis and Simulation of Wireless and Mobile Systems 2010. In 2009 he was designated IEEE Fellow for his contributions to the analysis and design of communication networks. He is on the technical advisory board of IMDEA Networks . 